Nervenarzt 2020 $91: 537-540$

https://doi.org/10.1007/s00115-020-00912-1

Online publiziert: 4. Mai 2020

(c) Der/die Autor(en) 2020

Tobias Meindl' • Isabell Cordts ${ }^{1}$ Anna-Lisa Scherzer' - Paul Lingor ${ }^{1}$ • Christian Maegerlein ${ }^{2}$. Valentina Galassi Deforie ${ }^{3}$. Natalia Dominik ${ }^{3}$. Henry Houlden ${ }^{3} \cdot$ Andrea Cortese $^{3,4} \cdot$ Marcus Deschauer $^{1}$

${ }^{1}$ Klinik und Poliklinik für Neurologie, Klinikum rechts der Isar, Technische Universität München, München, Deutschland

${ }^{2}$ Abteilung für Neuroradiologie, Klinikum rechts der Isar, Technische Universität München, München, Deutschland

${ }^{3}$ Department of Neuromuscular Disease, UCL Queen Square Institute of Neurology and The National Hospital for Neurology and Neurosurgery, London, Großbritannien

${ }^{4}$ Department of Brain and Behavioral Sciences, University of Pavia, Pavia, Italien

\title{
CANVAS: Fallbericht einer neuen Repeat-Erkrankung mit spät beginnender Ataxie
}

\section{Kasuistik}

Eine 74-jährige Patientin stellte sich wegen seit 14 Jahren bestehendem Schwindel vor. Seit 10 Jahren litt sie unter einer Gangunsicherheit sowie einem Taubheitsgefühl an Fingern und Zehen. Seit 2 Jahren klang die Sprache verwaschen. Alle Symptome waren langsam progredient. Beim Gehen wackelte das Bild und bei Augenschluss stürzte sie. Außerdem litt sie unter einem trockenen Husten, einer Motilitätsstörung des Ösophagus und Obstipation. Die Familienanamnese für ähnliche Beschwerden war leer.

In der körperlichen Untersuchung fand sich eine Zeigeataxie. Der Romberg-Versuch war unsicher, das Gangbild breitbasig. Es bestand eine skandierende Sprache. Die Blickfolge war sakkadiert. Der Kopf-Impuls-Test zeigte eine beidseitige vestibuläre Untererregbarkeit mit Einstellsakkaden. Berührungs- und Vibrationsempfinden sowie der Lagesinn waren an den Extremitäten distal beeinträchtigt. Der SARA-Score (Score of Assessment and Rating of Ataxia) betrug 9,5 Punkte.

Die kalorische Spülung wies eine bilaterale vestibuläre Untererregbarkeit nach. Eine Magnetresonanztomographie (MRT) des Kopfes zeigte eine besonders den Kleinhirnwurm betreffende zerebelläre Atrophie ( $\bullet$ Abb. 1$)$. Im spinalen MRT fand sich eine Myelonatrophie
(• Abb. 2). Die sensiblen Neurographien zeigten am N. suralis und N. medianus keine Reizantworten, am N. ulnaris eine leichte Amplitudenminderung. Die motorischen Neurographien des N. medianus, N. ulnaris, N. peroneus und $\mathrm{N}$. tibialis zeigten leicht verminderte Amplituden. Sensibel evozierte kortikale Potenziale waren nach Stimulation an $\mathrm{N}$. tibialis und N. medianus nicht ableitbar. Eine Hypovitaminose (Vitamin E, B6, B12) wurde ausgeschlossen. Liquordiagnostisch bestanden keine entzündlichen Veränderungen. Die genetische Diagnostik häufiger spinozerebellärer Ataxien (SCA1, 2, 3, 6, 7 und 17) sowie der Friedreich-Ataxie war negativ.

\section{Diagnose}

Bereits die Anamnese ergab Hinweise auf Beeinträchtigungen von Vestibularapparat und Kleinhirn sowie eine Polyneuropathie: Die Oszillopsien sprachen für eine bilaterale Vestibulopathie, die verwaschene Sprache für ein zerebelläres Syndrom, die akrale Taubheit und die Stürze bei Augenschluss für eine sensible, afferente, Ataxie bei Polyneuropathie. Alle drei Systeme fanden sich auch in der klinischen Untersuchung beeinträchtigt. Somit konnte die Syndromdiagnose CANVAS gestellt werden.

Mittels Repeat-primed-Polymerase-Kettenreaktion [2] im RFC1-Gen 

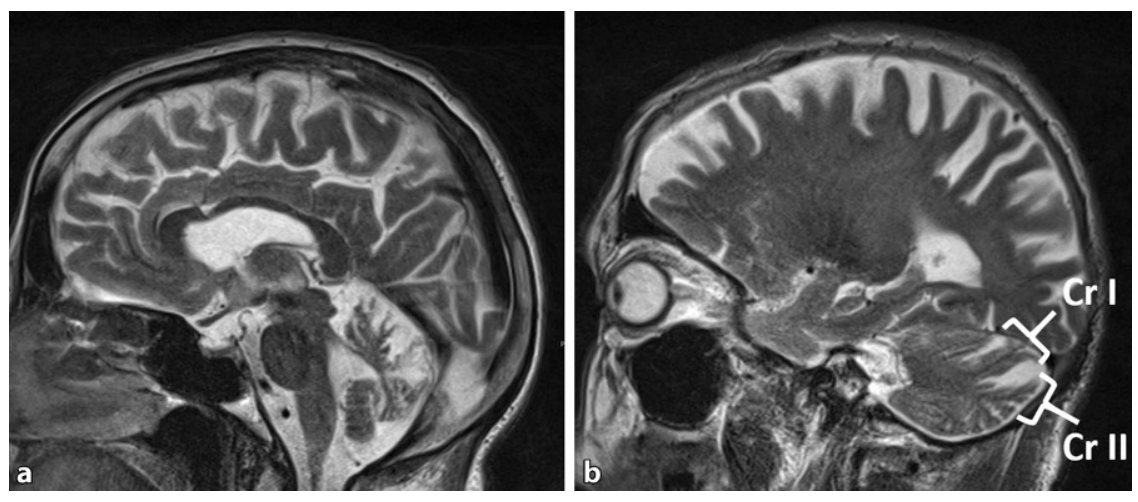

Abb. 1 ॥ T2-gewichtete sagittale (a) und parasagittale (b) zerebrale Magnetresonanztomographie: akzentuierte Atrophie des Vermis (a) sowie auch des Crus ( $\mathrm{Cr}$ ) I und II (b)
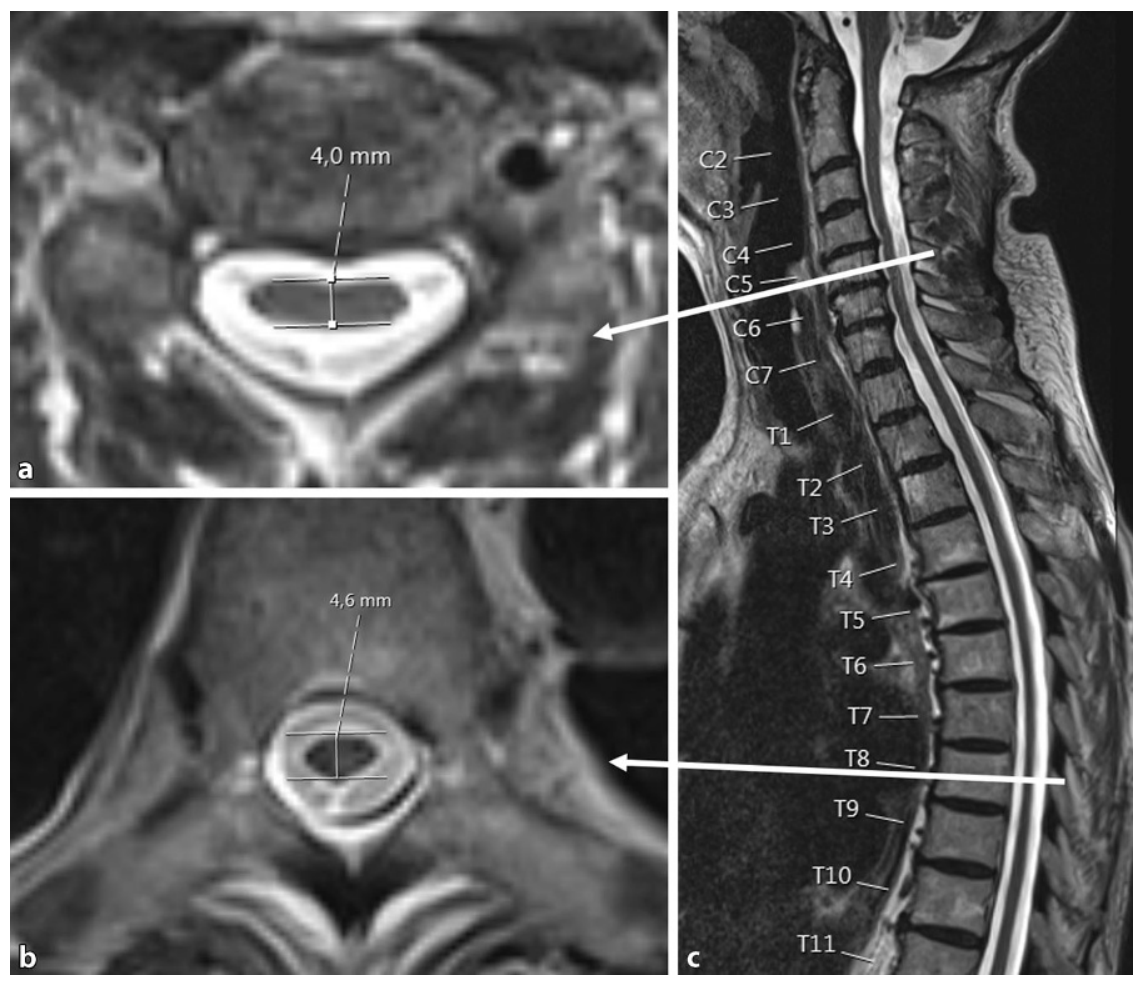

Abb. 2 ム T2-gewichtete spinale Magnetresonanztomographie sagittal (c) sowie axial in Höhe des Halswirbelkörpers (HWK) 5 (a) und Brustwirbelkörpers (BWK) 8 (b): langstreckige Myelonatrophie mit einem Tiefendurchmesser von 4,0 mm auf Höhe HWK 5 (normal 7,4 $\pm 1,6 \mathrm{~mm}$ [2 SDs]; [4]) und 4,6 mm auf Höhe BWK 8 (normal 6,3 $\pm 2,0 \mathrm{~mm}$ [2 SDs]; [4])

konnte auf beiden Allelen die pathologische AAGGG-Pentanukleotid-Expansion nachgewiesen werden, während nichtpathogene AAAGG- bzw. AAAAGExpansionen nicht vorlagen. Das Wildtypallel mit 11 AAAAG-Repeats war mittels flankierender PCR nicht nachweisbar.

\section{Diskussion}

Zerebelläre Ataxie, sensible Neuropathie und Vestibulopathie stellen die Kardinalsymptome des CANVAS dar, das 2011 erstmals als Syndrom beschrieben wurde [10]. Darüber hinaus können autonome Symptome wie chronischer Husten, Ösophagusmotilitätsstörung und Obstipation auftreten. Post-mortem-Untersuchungen an CANVAS-Patienten zeigten eine Atrophie der Hinterstränge infol- ge der Degeneration der Spinalganglien und einen Purkinje-Zell-Verlust vor allem im Vermis [8]. Die größte klinische Diagnosesicherheit erbrachte bisher der Nachweis einer vestibulären Untererregbarkeit in der Videookulographie oder Videonystagmographie, der bildgebende Nachweis einer im Vermis betonten zerebellären Atrophie und der elektrophysiologische Nachweis einer sensiblen Neuropathie bei molekulargenetischem Ausschluss häufiger hereditärer Ataxien [9]. Im Jahr 2019 wurde eine biallelische Expansion im RFC1-Gen, mit 400 bis 2000 AAGGG-Pentanukleotiden (statt 11 Repeats des AAAAG-Wildtypallels) als genetische Ursache identifiziert [2] und in zwei weiteren Arbeiten bestätigt $[1,6]$. Bei RFC1 handelt es sich um eine Untereinheit des RFC-Proteinkomplexes, der für die DNA-Replikation wichtig ist. Der genaue Pathomechanismus der Erkrankung ist noch unklar $[2,6]$.

Die Beschreibung von 100 Patienten mit genetisch gesicherter RFC1-Expansion zeigte ein medianes Erkrankungsalter von 52 Jahren (19-76 Jahre). Die sensible Neurographie war stets abnormal, eine zerebelläre Atrophie fand sich in $63 \%$ und eine Myelonatrophie in $45 \%$ der MRTs. Erstsymptom war häufig eine sensible Polyneuropathie, manchmal aber auch ein spasmodischer Husten. Zerebelläre Symptome (Dysarthrie) und vestibuläre Symptome (Oszillopsien) traten typischerweise später auf [3]. Bei unserer Patientin bestanden alle charakteristischen Symptome und auch die Ergebnisse der apparativen Diagnostik waren typisch.

Bei Patienten mit ätiologisch ungeklärter Ataxie ohne dem Vollbild eines CANVAS-Syndroms fand sich die Repeat-Expansion im RFC1-Gen bei $22 \%$ aller Patienten, $63 \%$ der Patienten mit Ataxie und Neuropathie und $92 \%$ der Patienten mit allen Kardinalsymptomen [2]. Die CANVAS-Häufigkeit wurde auf 5 bis 13/100.000 Personen geschätzt [1, $2,6]$.

Wegen der hohen "Trefferquote " unter Patienten mit spät beginnender Ataxie sowie dem mit $2 \%$ vergleichsweise hohen Anteil von CANVAS-Patienten in einer Kohorte deutscher Schwindelpatienten [7] sollte die Untersuchung der RFC1-Gen-Expansion bei allen Pati- 
enten mit einer nichterworbenen spät beginnenden Ataxie erfolgen, nicht nur bei autosomal-rezessivem Erbgang mit betroffenen Geschwistern, sondern auch bei sporadischen Fällen. Dabei ist zu berücksichtigen, dass die Expansion nur durch eine gezielte molekulargenetische Testung mittels PCR oder Southern-BlotAnalyse erkannt werden kann: Die intronische Expansion entgeht Gen-Paneloder Exomuntersuchungen. Aktuelle Methoden der Next-generation-Sequenzierung nämlich beruhen auf sog. „shortreads" um 150 Basenpaare. Daher sind sie nicht zum Nachweis von Expansionen von 2000 bis 10.0000 Basenpaaren, wie sie bei der Pentanukleotidexpansion im RFC1-Gen bestehen, geeignet.

\section{Fazit für die Praxis}

\section{- Eine Repeat-Expansion im RFC1-Gen liegt dem CANVAS zugrunde und stellt mutmaßlich eine häufige ge- netische Ursache spät beginnender Ataxien dar. \\ - Bei Patienten mit sporadischer spät beginnender Ataxie (5. bis 7. Le- bensjahrzehnt) ohne Hinweise auf eine erworbene Ursache sollte ei- ne Untersuchung des RFC1-Gens vorgenommen werden. \\ - Eine gezielte molekulargenetische Diagnostik, die mittlerweile auch in deutschen Laboren etabliert wurde, ist notwendig, da die Expansion Next-generation-Sequenzierungen entgeht.}

\section{Korrespondenzadresse}

\section{Dr. Tobias Meindl}

Klinik und Poliklinik für Neurologie, Klinikum rechts der Isar, Technische Universität München Ismaninger Str. 22, 81675 München, Deutschland

tobias.meindl@tum.de

Danksagung. Wir danken Dr. D. Gläser, Neu-Ulm und Dr. T. Haack, Tübingen für den Ausschluss anderer genetischer Ataxie-Ursachen.

Funding. Open Access funding provided by Projekt DEAL.

Nervenarzt 2020 $91: 537-540$ https://doi.org/10.1007/s00115-020-00912-1

(c) Der/die Autor(en) 2020

T. Meindl · I. Cordts · A.-L. Scherzer · P. Lingor · C. Maegerlein · V. Galassi Deforie · N. Dominik · H. Houlden $\cdot$ A. Cortese $\cdot M$. Deschauer

\section{CANVAS: Fallbericht einer neuen Repeat-Erkrankung mit spät beginnender Ataxie}

\section{Zusammenfassung}

Wir schildern den Fall einer 74-jährigen Patientin, die ab dem 60. Lebensjahr ein progressives Krankheitsbild mit sensibler Neuropathie, zerebellärer Ataxie und bilateraler Vestibulopathie entwickelte. Die Familienanamnese war leer. Magnetresonanztomographisch zeigten sich eine im Vermis betonte Kleinhirnatrophie sowie eine Myelonatrophie. Syndromal wurde ein CANVAS (zerebelläre Ataxie, Neuropathie, vestibuläre Areflexie Syndrom) diagnostiziert. Für diese Erkrankung wurde 2019 der Gendefekt beschrieben, eine autosomal-rezessiv vererbte intronische Repeat-Expansion im RFC1-Gen. Diese Veränderung war bei unserer Patientin biallelisch nachweisbar. CANVAS ist eine relativ häufige Ursache einer hereditären spät beginnenden Ataxie (geschätzte Häufigkeit 5-13/100.000). Nicht immer liegt wie bei unserer Patientin das Vollbild vor. Daher sollte bei Patienten mit unklarer sporadischer spät beginnender Ataxie die Untersuchung der RFC1-Gen-Expansion erwogen werden. Da die intronische Repeat-Expansion der Nextgeneration-Sequenzierung entgeht, ist eine gezielte Diagnostik erforderlich.

Schlüsselwörter

CANVAS - RFC1 - Repeat-Erkrankung · Ataxie Vestibulopathie · Polyneuropathie · Später Beginn

\section{CANVAS: case report on a novel repeat expansion disorder with late-onset ataxia}

\section{Abstract}

This article presents the case of a 74-yearold female patient who first developed a progressive disease with sensory neuropathy, cerebellar ataxia and bilateral vestibulopathy at the age of 60 years. The family history was unremarkable. Magnetic resonance imaging (MRI) showed atrophy of the cerebellum predominantly in the vermis and atrophy of the spinal cord. The patient was given the syndromic diagnosis of cerebellar ataxia, neuropathy, vestibular areflexia syndrome (CANVAS). In 2019 the underlying genetic cause of CANVAS was discovered to be an intronic repeat expansion in the RFC1 gene with autosomal recessive inheritance. The patient exhibited the full clinical picture of CANVAS and was tested positive for this repeat expansion on both alleles. The CANVAS is a relatively frequent cause of late-onset hereditary ataxia (estimated prevalence $5-13 / 100,000)$. In contrast to the present patient, the full clinical picture is not always present. Therefore, testing for the RFC1 gene expansion is recommended in the workup of patients with otherwise unexplained late-onset sporadic ataxia. As intronic repeat expansions cannot be identified by next generation sequencing methods, specific testing is necessary.

Keywords CANVAS - RFC1 - Repeat expansion disease . Ataxia - Vestibulopathy Polyneuropathy . Late onset

\section{Einhaltung ethischer Richtlinien}

Interessenkonflikt. T. Meindl, I. Cordts, A.-L. Scherzer, P. Lingor, C. Maegerlein, V. Galassi Deforie, N. Dominik, H. Houlden, A. Cortese und M. Deschauer geben an, dass kein Interessenkonflikt besteht.

Für diesen Beitrag wurden von den Autoren keine Studien an Menschen oder Tieren durchgeführt. Für die aufgeführten Studien gelten die jeweils dort angegebenen ethischen Richtlinien. Für Bildmaterial oder anderweitige Angaben innerhalb des Manuskripts, über die Patienten zu identifizieren sind, liegt von ihnen und/oder ihren gesetzlichen Vertretern eine schriftliche Einwilligung vor.

Open Access Dieser Artikel wird unter der Creative Commons Namensnennung 4.0 International Lizenz veröffentlicht, welche die Nutzung, Vervielfältigung, Bearbeitung, Verbreitung und Wiedergabe in jeglichem Medium und Format erlaubt, sofern Sie den/die ursprünglichen Autor(en) und die Quelle ordnungsge- 
mäß nennen, einen Link zur Creative Commons Lizenz beifügen und angeben, ob Änderungen vorgenommen wurden.

Die in diesem Artikel enthaltenen Bilder und sonstiges Drittmaterial unterliegen ebenfalls der genannten Creative Commons Lizenz, sofern sich aus der Abbildungslegende nichts anderes ergibt. Sofern das betreffende Material nicht unter der genannten Creative Commons Lizenz steht und die betreffende Handlung nicht nach gesetzlichen Vorschriften erlaubt ist, ist für die oben aufgeführten Weiterverwendungen des $\mathrm{Ma}$ terials die Einwilligung des jeweiligen Rechteinhabers einzuholen.

Weitere Details zur Lizenz entnehmen Sie bitte der Lizenzinformation auf http://creativecommons.org/ licenses/by/4.0/deed.de.

\section{Literatur}

1. Akçimen F, Ross JP, Bourassa CV et al (2019) Investigation of the RFC1 repeat expansion in a Canadian and a Brazilian ataxia cohort: identification of novel conformations. Front Genet 10:1219-1219

2. Cortese A, Simone R, Sullivan R et al (2019) Biallelic expansion of an intronic repeat in RFC1 is a common cause of late-onset ataxia. Nat Genet 51:649-658

3. Cortese A, Tozza S, Yau WY et al (2020) Cerebellar ataxia, neuropathy, vestibular areflexia syndrome due to RFC1 repeat expansion. Brain 143:480-490

4. Frostell A, Hakim R, Thelin EP et al (2016) A review of the segmental diameter of the healthy human spinal Cord. Front Neurol 7:238-238

5. Giordano I, Harmuth F, Jacobi H et al (2017) Clinical and genetic characteristics of sporadic adult-onset degenerative ataxia. Neurology 89:1043-1049

6. Rafehi H, Szmulewicz DJ, Bennett MF et al (2019) Bioinformatics-based identification of expanded repeats: a non-reference Intronic Pentamer expansion in RFC1 causes CANVAS. Am J Hum Genet 105:151-165

7. Strupp M, Dieterich M, Brandt T (2013) The treatment and natural course of peripheral and central vertigo. Dtsch Arztebl Int 110:505-516

8. Szmulewicz DJ, Mclean CA, Rodriguez ML et al (2014) Dorsal root ganglionopathy is responsible for the sensory impairment in CANVAS. Neurology 82:1410-1415

9. Szmulewicz DJ, Roberts L, Mclean CA et al (2016) Proposed diagnostic criteria for cerebellar ataxia with neuropathy and vestibular areflexia syndrome (CANVAS). Neurol Clin Pract 6:61-68

10. Szmulewicz DJ, Waterston JA, Halmagyi GM et al (2011) Sensory neuropathy as part of the cerebellar ataxia neuropathy vestibular areflexia syndrome. Neurology 76:1903-1910

In eigener Sache

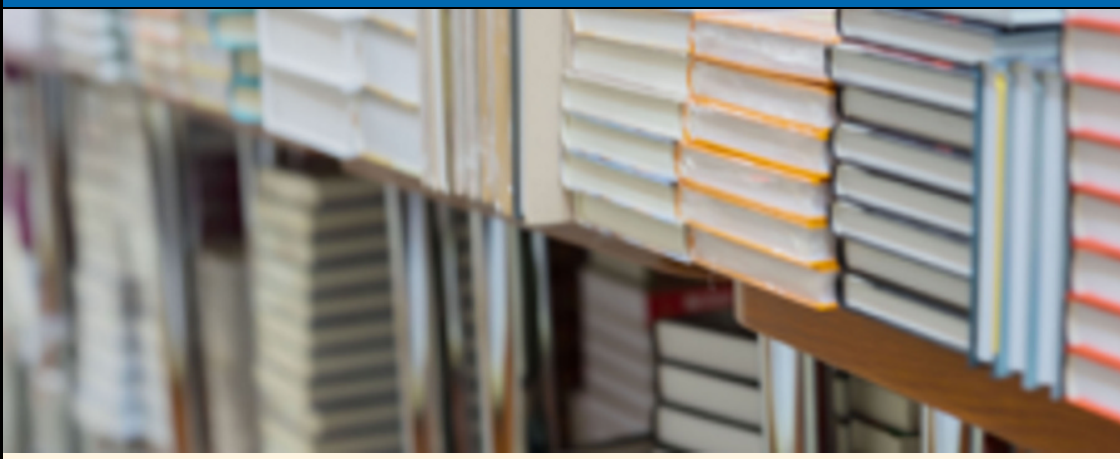

Springer Nature stellt wichtige Lehrbücher während der CoronaKrise kostenfrei zur Verfügung

\section{Lehrbuchpaket zur Intensivmedizin und Pneumologie für pflegerisches und medizinisches Fachpersonal}

Der Ausbruch des Coronavirus bedeutet nachhaltige Einschränkungen für den wissenschaftlichen Lehrbetrieb. Um die nachteiligen Auswirkungen zu lindern, startet Springer Nature ein globales Programm zur Unterstützung des Lernens und Lehrens in Hochschuleinrichtungen weltweit. Lehrende und Studierende aus akademischen Einrichtungen können kostenlos auf mehr als 500 wichtige englisch- und deutschsprachige Lehrbücher von Springer Nature zugreifen. Diese Bücher sind voraussichtlich bis mindestens Ende Juli über die Plattform SpringerLink zugänglich (URL: siehe „Weitere Informationen" am Ende dieser Mitteilung).

Springer Nature hat bereits Tausende von Forschungsartikeln, Büchern und Buchkapiteln zum Thema Coronavirus frei verfügbar gemacht, um den Zugang zu wichtigen Forschungsergebnissen zu erleichtern. Bibliothekare werden unterstützt, wenn sie Mitarbeitern und Studenten einen OnlineZugriff von zuhause ermöglichen möchten. Diese neue Lehrbuch-Initiative zielt darauf ab, das Lernen und Lehren effizient zu unterstützen. Studenten, Akademiker und Universitätsmitarbeiter haben nun freien Zugang zu wichtigen Springer Nature-Lehrbüchern.

Niels Peter Thomas, Managing Director Springer Nature Books: „Angesichts der zunehmenden globalen Auswirkungen der CoronaKrise ist der schnelle Fernzugriff auf Bildungsressourcen unerlässlich geworden. Wir möchten Dozenten, Lehrer und Studenten in dieser herausfordernden Zeit unterstützen und hoffen, dass wir mit der kostenlosen
Freischaltung von über 500 wichtigen Lehrbüchern dazu einen wichtigen Beitrag liefern. An dieser Stelle möchte ich mich für die großartige Unterstützung durch unsere Autoren und Autorinnen bedanken, ohne deren $\mathrm{Zu}$ stimmung wir diese Bücher nicht so ohne weiteres hätten freischalten können."

Ein Buchpaket mit deutschsprachigen Lehrund Fachbüchern für pflegerisches und medizinisches Fachpersonal aus den Gebieten Intensivmedizin und Pneumologie wird ebenfalls auf der Plattform SpringerLink freigeschaltet. Dies ist ein kostenloses Angebot für klinische Einrichtungen in der aktuellen Ausnahmesituation: Der Wissenschaftsverlag möchte Krankenhäusern dabei helfen, sein Personal so fortzubilden, dass es ausreichend qualifiziert ist, um die vermehrt erwarteten Corona-Patienten mit schwerem Verlauf kompetent zu versorgen.

\section{Weitere Informationen:}

Zugang zu den freigeschalteten Lehrbüchern (alle Disziplinen):

www.springernature.com/gp/librarians/newsevents/all-news-articles/industry-newsinitiatives/free-access-to-textbooks-for-institutions-affected-by-coronaviru/17855960

Übersicht aller freigeschalteten Zeitschriftenartikel, Kapitel und Bücher zur CoronavirusForschung: www.springernature.com/de/researchers/ campaigns/coronavirus

\section{Zur Plattform SpringerLink: link.springer.com}

